\title{
Tranexamic acid use and preoperative haemoglobin level predict perioperative red blood cell transfusions in hip arthroplasty
}

C. Campos ${ }^{1}$, J. Calheiros ${ }^{1}$, R. Fernandes ${ }^{1}$, C.Pinto $^{1}$, S. Vide ${ }^{1}$

1 ULSM-HPH, Department of Anesthesia - Matosinhos (Portugal),

Background: There is a high prevalence of blood product transfusions among patients undergoing total hip replacement surgery.(1) Preoperative estimation of transfusion may be useful to optimize perioperative blood management.(2) The simplest methods to do this are those that identify patients at risk of requiring transfusion support on the basis of the surgical and few clinical parameters.(2) The aim of this study was to assess if we could find in our clinical practice predictors of transfusion.

Methods and Materials: This is an observational retrospective study where 178 patients undergoing elective hip arthroplasty were considered. Demographic data (age and sex) as well as preoperative haemoglobin and use of tranexamic acid were included into a logistic regression for perioperative red blood cell transfusion. Data are presented as mean \pm standard deviation.
Results and Discussion: The logistic regression model showed goodness of fit (Hosmer and Lemeshow test $p=$ 0,953).

Preoperative haemoglobin levels and the use of tranexamic acid were statistically significative $(p<0,001$, $\operatorname{Exp}(B) 0,412$ and $p=0,043, \operatorname{Exp}(B) 0,222$ respectively).

ROC curve of the predicted probabilities yielded an AUC of 0,852 $(p<0,001)$.

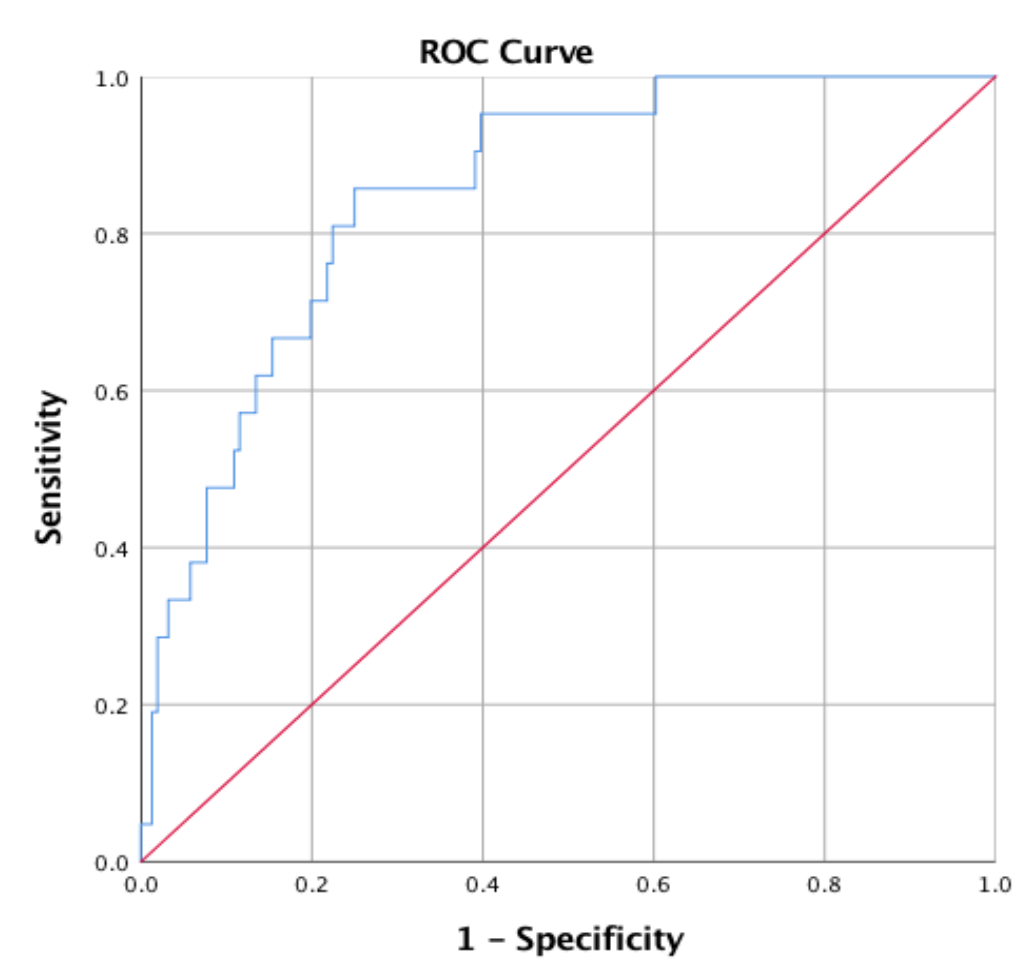

Conclusions: It seems that variables like
preoperative haemoglobin and the use of
tranexamic acid, might be able to predict the
perioperative requirement for transfusion of red
blood cells.

